\title{
Comparison of Methods in Studies of Cell Death Mechanisms
}

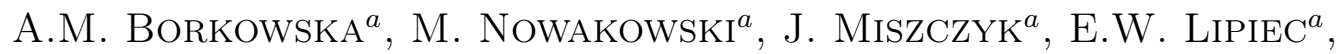 \\ J. Wiltowska-Zuber ${ }^{a}$, K. RAWOJĆ ${ }^{b}$ AND W.M. KWIATEK ${ }^{a, *}$ \\ ${ }^{a}$ Institute of Nuclear Physics, Polish Academy of Sciences, PL-31-342 Krakow, Poland \\ ${ }^{b}$ Department of Endocrinology, Nuclear Medicine Unit, The University Hospital, Kraków, Poland \\ While studying the influence of ionizing radiation or certain chemical agents on cells, it is crucial to not only \\ determine cytotoxicity, but also to follow cell death mechanisms. There are different methods to screen processes of \\ cell death and still very important question remains unanswered about differences in results that could be caused \\ by various experimental steps in procedures. Based on literature review two protocols of cell death determination \\ were compared. First protocol regarded collecting cells floating in medium before trypsinization and following \\ centrifugation of them. In the second protocol floating cells were discarded and attached ones were stained and \\ fixed. In all experiments three different untreated cell lines (A172, DU145 as cancer cell lines and in comparison, \\ fibroblasts (FB CCL 110), as a non- cancerous cell line) were used to test applied protocols. Cells were cultured \\ and death processes were examined at different time points up to $120 \mathrm{~h}$. Compared protocols showed statistically \\ significant differences, especially in terms of necrosis, which was higher when included floating cells from culture \\ medium and then centrifuging them. Therefore, presented results show importance of choosing a valid experimental \\ procedure in case of evaluating cells viability and types of cell death pathways quantitatively.
}

DOI: 10.12693/APhysPolA.133.263

PACS/topics: growth and division, cell processes, fluorescence, optimization

\section{Introduction}

Determination of cellular death type is crucial in investigating the influence of damaging factors such as radiation or chemical cytotoxic agents on cells. Various methods and protocols are used to determine type of cellular death. Following the Nomenclature Committee on Cell Death (NCCD) recommendations, a unified criteria to define cell death type were proposed $[1,2]$. Based on the morphological differences, one can distinguish two major types of cell death: apoptosis and necrosis [2]. According to NCCD, apoptosis is characterized by rounding-up of the cell, reduction of cellular volume, chromatin condensation, nuclear fragmentation and engulfment by resident phagocytes, thus preventing them from the release of cellular debris and inflammation [2,3]. On the contrary to apoptosis, necrosis is characterized by increase of cell volume, cytoplasm and cytoplasmic organelle swelling, moderate chromatin condensation, plasma membrane rupture and loss of intracellular contents. This causes the induction of inflammatory response and potentially local immune activation $[2,3]$. Application of some experimental procedures in vitro can lead to decreased cell viability and increased level of dead cells. Cell culture parameters, i.e. chosen variant of cell harvesting, either by mechanical scrapping or by enzymes and centrifugation may lead to false positive or false negative results [4-10].

The awareness of differences in the results when using different protocol variants, based on the same molecular method is crucial especially during quantitative studies.

*corresponding author; e-mail: Wojciech.Kwiatek@ifj.edu.pl
Therefore, the idea of this paper is to compare two the most frequently used protocol variants to detect apoptosis and necrosis based on well-known fluorescence staining and trypan blue techniques. This comparison can show if there are significant differences in the fraction of non-viable cells and those undergoing through apoptosis and necrosis.

\section{Methods}

\subsection{Cells culturing}

Three cell lines were used in the experiment. Two were cancer cell lines: A172 (human brain glioblastoma), DU145 (human prostate cancer, brain metastasis) and for comparison normal non-cancerous fibroblasts (FB CCL 110) were applied. All types of cell lines with similar passage number (no. 4 for A172 and fibroblast cells and no. 5 for DU145 cells) were seeded into 24-well plates (NEST) in number of $10^{4}$ cells/well. Each experiment was repeated twice independently. After seeding, cells were appropriately supplemented according to protocols provided by ATCC Organization and incubated at $37^{\circ} \mathrm{C}$ in a humidified atmosphere incubator containing $5 \% \mathrm{CO}_{2}$ for $24,48,72,96$, and $120 \mathrm{~h}$. For studies of cell proliferation and cell death mechanisms using method with trypsinization, cells were seeded directly into wells. In protocol variant without trypsinization step, cells were seeded into sterile cover glasses (Sarstedt, $11 \mathrm{~mm}$ ).

\subsection{Cell proliferation analysis}

To analyse proliferation and to detect non-viable cells in population, cells were stained by routinely used trypan blue dye (Sigma Aldrich, final concentration up to $0.2 \%$, dissolved in PBS) [11]. At each time point the number 
of living and dead cells was determined using a Bürker chamber. The doubling time for each cell line was calculated using assumption of cell population's exponential growth $[12,13]$. Therefore, logarithm of cell number was calculated and then growth rate was obtained using linear fitting. Doubling time was finally calculated using Eq. (1):

$$
t_{D}=\frac{\ln 2}{a},
$$

where $t_{D}$ is doubling time, while $a$ is growth rate of cells in population.

\subsection{Fluorescent staining}

To quantify both types of cells death, a solution of fluorescent dyes in binding buffer $(1 \times)$ was used. This allowed simultaneous quantification of apoptotic and necrotic cells by fluorescence microscopy. The Hoechst $33258(10 \mu \mathrm{g} / \mathrm{ml})$ was used to identify all cells in a population, propidium iodide $(\mathrm{PI}, 1 \mathrm{\mu g} / \mathrm{ml}$ ) was added to observe fraction of necrotic cells and Annexin V FITC Conjugate $(0.25 \mu \mathrm{g} / \mathrm{ml})$ was used to determine fraction of cells in apoptotic state. Cells stained by both PI and Annexin V were considered as apoptotic ones and were subtracted from fraction of necrotic cells. PI is routinely used to stain DNA and detect dead cells because of its ability to penetrate cell membrane only when it is permeable regardless of the mechanism of death [14]. On the other hand, Annexin V might be applied to distinguish apoptotic cells from necrotic ones based on changes in cell membrane caused by apoptosis [4]. During apoptosis phosphatidylserine (PS) is exposed at the cell surface and Annexin V has high affinity to PS. Annexin V cannot penetrate cell membrane and therefore it can be used for apoptosis detection [4].

In the first protocol variant (denoted further as pI), trypsinization of cells was performed. Additionally, cells floating in culture medium were collected to determine fraction of living, apoptotic and necrotic ones. Cells were centrifuged (1600 rpm, $5 \mathrm{~min}$ ), supernatant was removed and finally $30 \mathrm{\mu l}$ of solution of fluorescent dyes in binding buffer $(1 \times)$ was added. In the second protocol variant (denoted further as pII), dead cells floating in culture medium were removed and living cells were left adhered to cover glasses. Following, cells were washed using PBS buffer (Sigma Aldrich). In this variant, neither trypsinization nor centrifugation procedures were applied. At the end, all cells regardless of the protocol, were stained using $30 \mu \mathrm{l}$ of fluorescent dyes solution in binding buffer $(1 \times)$.

After 10 min of cells incubation with fluorescent dyes (in darkness), images were captured using fluorescent microscope (Olympus BX51, $100 \mathrm{~W}$ mercury lamp, 4×). In case of not trypsinized cells, they were washed using binding buffer and fixed using paraformaldehyde for $10 \mathrm{~min}$ (3.7\%, Sigma Aldrich) prior to image collection. Obtained photos were analysed using ImageJ software (NIH Image, open source) and for each set of photos fraction in percent of living, apoptotic and necrotic cells was cal- culated. From each well, photos of at least three different areas were analysed. This gave at least nine areas of interest for each experimental point. Therefore, total number of cells per one experimental point was between 60 and 8900 depending on the cell line, time point and chosen protocol variant.

\subsection{Statistical analysis}

Results are presented as mean \pm standard deviation (SD) in percentages. Statistical analysis was performed using Statistica 10 with a 0.05 cut-off for each analysis. Variables were tested for normality by the Shapiro-Wilk test and also graphs of normality were plotted. Due to having two distribution types: normal and nonparametric, statistical analyses were used for determining statistical significance. To study changes between independent groups for two different methods of cell death type determination, the Mann-Whitney U Test was used. On the other hand, to study changes between dependent groups for two time points Wilcoxon paired signed-rank test was performed. In order to compare doubling time between cell lines, $t$-Student test was used $(p<0.05)$.

\section{Results and discussion \\ 3.1. Determination of doubling time and population of living cells}

For A172, DU145 and fibroblast cells, doubling time $\left(t_{D}\right)$ of cell population was calculated based on linear fitting to the logarithm of cell's number (Fig. 1). Obtained results were as follows: $t_{D}=28.2 \pm 4.1 \mathrm{~h}$ for A172 cell line, $t_{D}=23.1 \pm 1.6 \mathrm{~h}$ for DU145 cells and $t_{D}=31.4 \pm 5.0 \mathrm{~h}$ for fibroblasts (Fig. 1b,d,f). $t_{D}$ obtained for A172 cell line was statistically different $(p<0.05)$ from $t_{D}$ obtained for DU145 cells but not for fibroblasts. Additionally, $t_{D}$ for DU145 cells was significantly different $(p<0.05)$ in comparison to doubling time for fibroblasts. Depending on protocol variant, averaged fraction of living cells in population was approximately estimated. In case of $\mathrm{pI}$ method, it was approximated in average by $97.2 \%$. On the other hand, for pII living cell fraction was approximated in average by $99.4 \%$. When compared fraction of living cells obtained using two protocols for different cell lines at applied time points, significant differences between pI and pII were observed. Time points at $t=96 \mathrm{~h}$ for A172 cell line and $t=24,72 \mathrm{~h}$ for fibroblasts were an exception. Observed tendencies are in accordance with results published by Batista et al. [5]. They observed damage of cell membrane after mechanical scrapping and highest cell viability after using trypsinization step for cell harvesting [5]. Additionally, Sutradhar et al. demonstrated that overtrypsinization severely affects cell membrane structure and may significantly reduce cell viability [6]. In our experiments, trypsin concentration and incubation time was experimentally confirmed with cell culture guidelines given by ATCC Organization. Therefore, used time was appropriate and did not lead to overtrypsinization. 

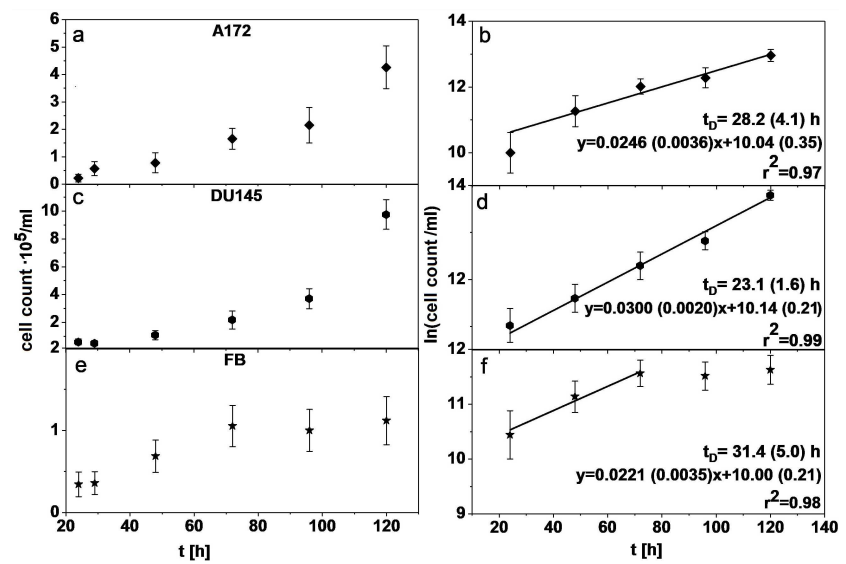

Fig. 1. Number of A172 (a), DU145 (c) and fibroblast (e) cells in function of time and calculated logarithm with line fitting $((\mathrm{b}),(\mathrm{d}),(\mathrm{f}))$.

\subsection{Influence of proposed methods on cell death type determination}

\subsubsection{Necrosis}

When compared two proposed methods for fraction's identification of living, necrotic and apoptotic cells in cell population, it was observed that there are significant differences between applied variants. When used pI variant, percent of necrotic cells was significantly higher at measured time points both for cancer and non- cancerous cell line $(p<0.05)$ in comparison to pII. Moreover, during experiment, fraction of cells undergoing through necrosis was similar for all studied cell lines. Time points at $t=72 \mathrm{~h}$ for A172 cell line and $t=120 \mathrm{~h}$ for fibroblasts were an exception. Additionally, for pI method, necrosis was approximately on averaged level of $1.9 \%$. In contrast, for pII variant, necrosis was approximately on averaged level of $0.1 \%$. Percent of cells undergoing through necrosis was similar for all studied cell lines in this method, however, differences in fractions of necrotic cells for both protocols used were statistically significant $(p<0.05)$. Observed differences might be caused by several reasons, but mostly, as we proposed, by application of trypsinization to detach cells from the flask surface and centrifugation. Different ways of cells detachment can lead to different levels of membrane damage and therefore can influence the percent of necrotic and apoptotic cells in population [4]. Bundscherer et al. concluded that trypsinization is a gentle method for cell detachment, but can be inadequate for example for metabolomic studies [4]. During centrifugation, some fraction of cells could be lost by removal of unpelleted ones in the supernatant [8]. Moreover, some fraction of cells are damaged after the centrifugation due to packing or repipetting them [8]. Katkov and Mazur pointed out that closely packed cells in the pellet might be probably more damaged during resuspension procedure [8]. Additionally, centrifugal damage of cells can be also caused by production of reactive oxygen species in the pellet [8]. Those damages can lead to decreased cell viability and increased cell death $[7,8]$.
Wang et al. presented that Burkitt lymphoma cells exposed to gravitational forces, recalculated to frequencies in the range of $14400-18700 \mathrm{rpm}$ at $25^{\circ} \mathrm{C}$ significantly lost their viability [9]. On the other hand, Son et al. proved that centrifugation with frequencies in the range of 1000-4000 rpm applied for 3 min has no influence on percentage of adipocytes and adipose stem cell viability [10]. Therefore, we postulate that centrifugation itself or further resuspension of cell's pellet may lead to cell damage and increase percentage of the necrotic cells in population. Moreover, it should be mentioned that studied cell lines can response differently for chosen centrifugal forces because of morphological and biological differences. When dying, adherent cell rounds up, detaches from the flask surface and floats in medium [15]. Therefore, for those kinds of cell lines, the most significant impact on the determined percent of apoptotic and necrotic cells in cell population has collection floating cells and taking them into consideration in further analysis. Despite the fact that the difference between $\mathrm{pI}$ and pII protocol in fraction of necrotic cells is significant, approximated level of $1.9 \%$ (pI) or $0.1 \%$ (pII) can be considered as a limit of statistical error in biological studies. Presented in the literature results of necrotic cells in fraction assessed by different methods depend on the cell treatment. What is more, they show statistical errors at the level ranging from a few percent up to several dozen of percents [16-18]. Therefore, effect of application of different protocols to determine cell death is important, but it not always must be critical for the obtained results. Time-dependent tendencies were observed in percentage of necrotic cells in population when pI variant was applied (Fig. 2). Therefore, changeability in percent of necrotic cells over time could be described by the Gauss function with centre of peak for A172, DU145 and fibroblast cells at $64.1 \pm 8.8 \mathrm{~h}\left(r^{2}=0.79\right.$, Fig. $\left.2 \mathrm{a}\right), 65.0 \pm 28 \mathrm{~h}$ $\left(r^{2}=0.52\right.$, Fig. 2b), 65.0 $\pm 11.0 \mathrm{~h}\left(r^{2}=0.68\right.$, Fig. 2c), correspondingly. On the other hand, good description of the change in the data over time was given by sinusoidal function $\left(r^{2}>0.8\right.$, Fig. 2), but the experiment should be conducted longer to prove this hypothesis. In case of pII method no time-dependent tendencies were observed and fraction of necrotic cells was constant.

\subsubsection{Apoptosis}

Analysis and comparison of results obtained for two applied protocol variants has shown that percent of apoptotic cells in cell population was similar in most cases. Following time points were an exception: $24 \mathrm{~h}$ for DU145 cells, $48 \mathrm{~h}$ for A172 and fibroblast cells and $120 \mathrm{~h}$ for DU145 cells. Moreover, approximated level of apoptosis was on averaged level of $1 \%$ in case of pI method applied and $0.5 \%$ when pII variant was used. Additionally, when analysed apoptotic fraction of cells for A172 and DU145 cell lines obtained with the use of pI variant it was exponentially decreasing with time constant equal to $18.7 \pm 8.0 \mathrm{~h}\left(r^{2}=0.91\right.$, Fig. $\left.2 \mathrm{a}\right)$ and $9.6 \pm 3.0 \mathrm{~h}$ $\left(r^{2}=0.94\right.$, Fig. 2b), respectively. On the other hand, in case of fibroblasts, tendency in decrease of apoptotic 


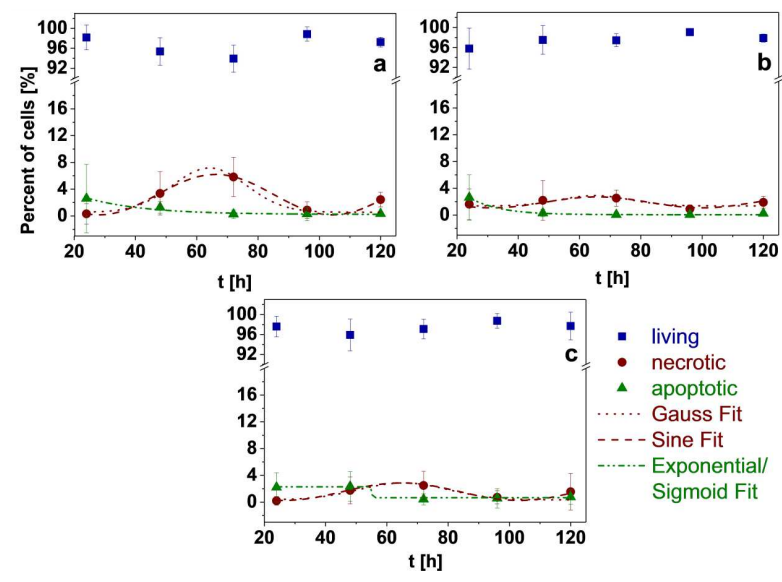

Fig. 2. Percent of living, necrotic, and apoptotic cells determined by protocol variant with trypsinization and centrifugation step applied $(\mathrm{pI})$ at the following time points: 24, 48, 72, 96, and $120 \mathrm{~h}$ for (a) A172 cells, (b) DU145 cells, and (c) fibroblasts with Gauss and sine function fit for necrotic cells and exponential or sigmoid function fit for cells undergoing through apoptosis.

cells could be described by sigmoid function centred at $55.0 \pm 9.2 \mathrm{~h}\left(r^{2}=0.92\right.$, Fig. 2c).

Bundscherer et al. demonstrated the influence of trypsinization and mechanical harvesting of cells on Annexin $\mathrm{V}$ staining and its influence of apoptosis determination [4]. Authors showed that in case of HT 29, PANC 1 and A-673 cell lines, a high percentage of cells were stained positively for Annexin $\mathrm{V}$ after mechanical detachment [4]. Moreover, detachment of cell could be also cause of apoptotic cell death called anoiks [2]. When this fraction of cells is removed, information about dead cells fraction is irrevocably lost. Application of this step in that cell death type determination could have extremely significant influence on obtained results.

\section{Summary}

We have demonstrated that the application of trypsinization, centrifugation and collection of floating in culture medium cells ( $\mathrm{pI}$ variant) has significant impact on increased fraction of necrotic cells and decreased fraction of living ones in population, but not on apoptotic subpopulation. Problem of cell death is crucial to understand effects of the influence of drugs or ionizing radiation on cells and following on tissues and organisms. Therefore, we find awareness of the influence of chosen protocol variant on obtained results. In further deduction and interpretation it is important to take into consideration application of certain experimental steps. It is of a high importance when one compare results between similar experiments.

\section{Acknowledgments}

This research project has been financed by the funds from the National Science Centre (Poland) granted on the basis of decision no. 2014/13/D/NZ1/01014. It was performed using equipment purchased in the frame of the project co-funded by the Małopolska Regional Operational Programme Measure 5.1 Krakow Metropolitan Area as an important hub of the European Research Area for 2007-2013, project No. MRPO.05.01.00-12013/15. Professor Małgorzata Lekka is gratefully acknowledged for providing A172 and DU145 cells for the experiment. Authors would like to thank for funding from Marian Smoluchowski Kraków Scientific Consortium "Mater-Energy-Future" under the KNOW grant.

\section{References}

[1] S. Orrenius, P. Nicotera, B. Zhivotovsky, Toxicol. Sci. 119, 3 (2011).

[2] G. Kroemer et al., Cell Death Differ. 16, 3 (2009).

[3] M.J. Berridge, Cell Signal. Biol. 6, csb0001011 (2014).

[4] A. Bundscherer, M. Malsy, R. Lange, P. Hofmann, T. Metterlein, B.M. Graf, M. Gruber, Anticancer Res. 33, 3201 (2013).

[5] U. Batista, M. Garvas, M. Nemec, M. Schara, P. Veranic, T. Koklic, Cell Biol. Int. 34, 663 (2010).

[6] B.C. Sutradhar, J. Park, G. Hong, S.H. Choi, G. Kim, Pak. Vet. J. 30, 232 (2010).

[7] I.I. Katkov, P. Mazur, Cell Biochem. Biophys. 31, 231 (1999).

[8] I.I. Katkov, P. Mazur, I.I. Katkov, J. Androl. 19, 232 (1998).

[9] D.I.C. Wang, T.J. Sinskey, R.E. Gerner, R.P. De Filippi, Biotechnol. Bioeng. 10, 641 (1968).

[10] D. Son, T. Choi, H. Yeo, J. Kim, K. Han, Ann. Plast. Surg. 72, 589 (2014).

[11] W. Strober, Curr. Protoc. Immunol. 111, 1 (2015).

[12] F. Widdel, Grundpraktikum Mikrobiol. 4, Universität Bremen, Bremen 2007.

[13] B.G. Hall, H. Acar, A. Nandipati, M. Barlow, Mol. Biol. Evol. 31, 232 (2014).

[14] L.C. Crowley, A.P. Scott, B.J. Marfell, J.A. Boughaba, G. Chojnowski, N.J. Waterhouse, Cold Spring Harb. Protoc. 2016, 647 (2016).

[15] O. Kepp, L. Galluzzi, M. Lipinski, J. Yuan, G. Kroemer, Nat. Rev. Drug Discov. 10, 221 (2011).

[16] A.M. Chinnaiyan, U. Prasad, S. Shankar, D.A. Hamstra, M. Shanaiah, T.L. Chenevert, B.D. Ross, A. Rehemtulla, Proc. Natl. Acad. Sci. USA 97, 1754 (2000).

[17] T. Hietanen, M. Pitkänen, M. Kapanen, P.L. Kellokumpu-Lehtinen, Int. J. Radiat. Biol. 92, 71 (2016).

[18] Y. Zhang, J. Guo, Y. Qi, Q. Shao, J. Liang, J. Radiat. Res. Appl. Sci. 7, 274 (2014). 